\title{
Characteristics of Corporate Network Structure Holes and Corporate Mergers and Acquisitions
}

\author{
Zhixing Lin
}

\author{
PANYAPIWAT INSTITUTE OF MANAGEMENT \\ Nonthaburi, Bangkok, Thailand \\ Email: $m f q b @ q q . c o m$
}

\begin{abstract}
This article is based on the theory of structural holes, based on the company network formed by the company's senior management team serving and taking part, and empirically studied the impact of the structural hole characteristics of Chinese listed company network on corporate mergers and acquisitions. The study found that because the company has more structural holes in the company network, it helps the company gain information advantages and thus obtain more $M$ \& A opportunities. The richness of the structural holes owned by the company has a significant positive correlation with the $\mathrm{M} \& \mathrm{~A}$ intentions. This article studies the social network and corporate mergers and acquisitions from the perspective of the company network formed by the senior management team serving and taking part in the job, which enriches the research results in related fields.

Keywords: social network, corporate network, structural hole, corporate mergers and acquisitions
\end{abstract}

\section{公司网络的结构洞特征与公司并购行为}

\author{
林枝兴
}

泰国正大管理学院, 暖武里, 曼谷, 泰国

$m f q b @ q q . c o m$

\begin{abstract}
摘要
本文基于结构洞理论, 以公司的高层管理团队任职兼任形成的公司网络为基础, 实证研究了中国上市 公司公司网络的结构洞特征对公司并购行为的影响。研究发现, 由于公司在公司网络中拥有更多的结 构洞, 有助于公司获得信息优势, 从而获得更多的并购机会, 公司拥有的结构洞丰富程度与并购意愿 呈显著正相关关系。本文从高层管理团队任职兼任形成的公司网络的视角研究社会网络与公司并购, 丰富了相关领域的研究成果。
\end{abstract}

关键词: 社会网络，公司网络，结构洞，公司并购

\section{1. 前言}

高层管理团队形成一个小团体，当这个小团体内部 的成员在其他公司任职时, 就会通过这种连锁任职形成 公司间的社会网络, 即公司网络。Haunschild（1994） 研究了具有连锁董事职位的公司, 并发现公司的并购决 定跟随公司董事兼任情况 [1]。整个上市公司构成的公 司网络中因所处的网络位置不同形成了每个公司之间的 结构洞, 而拥有高管兼任的公司将因其在公司网络中占 据了结构洞位置而享有信息优势和控制优势 (谢仁德和 陈运森, 2012） [2] 。因此, 基于结构洞理论, 采用结 构洞丰富程度将能够一定程度上反映公司网络对公司并 购决策的影响。公司的社会网络位置及其拥有的结构洞
丰富程度会如何影响公司的并购行为, 是一个值得研究 的问题。

在前人的研究中, 公司网络拘泥于通过连锁董事(董 事联结）构建董事网络, 实际上非董事在其他上市公司 兼职的现象也很普遍，公司与公司之间的社会网络的构 建, 有必要扩大至所有高管, 即由董事联结扩大至高管 联结。本文从高层管理团队任职兼任形成的公司网络的 视角研究社会网络与公司并购, 丰富了相关领域的研究 成果。

\section{2. 理论分析与研究假设}

1992 年美国社会学家 Ronald S. Burt 在《结构洞: 竞争的社会结构》一书中正式提出了结构洞理论 (Structural Holes Theory) [3] 。伯特认为, 结构洞能 
够为其占据者获取 “信息利益”和 “控制利益”提供机 会，从而比网络中其他位置上的成员更具有竞争优势。 董事联结在并购双方管理层之间构建了信息传递通道, 有助于降低并购方的搜寻、谈判成本，从而产生更大的 协同效应 (Cai and Sevilir，2012) [4]。Boussard 和 Godechot (2016) 研究了法国市场的公司并购, 发现公司 间的既有联系有助于并购市场的信任和交易 [5]。 Shipilov 和 Li (2008) 以社会网络结构为出发点, 认为占 据结构洞位置企业的边界取决于企业充分驾驭不断扩展 的网络的能力上限 [6]。万良勇、郑小玲（2014）曾以 结构洞理论为基础，对我国上市公司并购行为与结构洞 位置关系进行实证研究，研究发现在上市公司中，结构 洞越丰富, 并购方的相关董事掌握并购信息的优势越大, 这可以促使并购方提高并购行为质量, 促进并购行为的 开展 [7]。

在企业搜寻并购目标的过程中，高管联结无疑是直 接而快速的通道, 高管联结形成的公司网络中公司的结 构洞越丰富, 越能影响到企业高管团队的社会资本总量 与结构, 同时也影响到企业自身的社会资本，从而影响 到公司并购行为, 因此本文提出研究假设:

$\mathrm{H} 1$ : 其他条件不变的情况下, 上市公司在社会网络中 拥有的结构洞丰富程度与公司并购意愿具有正相关关系

\section{3. 研究设计}

\section{1. 样本选取与数据处理}

本文以 2008 2017 年间沪深两市A股上市公司发 起的并购事件为基本研究样本, 主要使用国泰安 (CSMAR) 数据库获得数据。高管联结数据通过查询国泰安数据库 高管兼任信息获得。

学者们认为, 社会网络关系正式形成后才能对并购 产生一定的影响（田高良等，2013）［8］。本文确定并 购事件的样本区间为 $2008 \sim 2017$ 年，高管网络数据样 本区间为 $2007 \sim 2016$ 年, 共 10 年, 样本按照如下原则 进行篮选:

（1）选择主并方为上市公司的并购样本；

（2）剔除金融行业的上市公司;

（3）择上市公司资产收购、股权转让和吸收合并等 狭义类型的并购行为剔除业务类型为债务重组的并购样 本, 剔除重组类型为资产剥离、债务重组、资产置换、 股份回购的并购样本；

（4）剔除交易当年处于 ST 类和终止上市类的公司;

(5) 将分次购入目标公司股权以实现并购视为一次 并购事件 ;

（6）剔除缺失 “重大资产重组标识”、“关联交 易标识”的并购交易

（7）剔除关联并购样本；

（8）剔除房地产公司购买地产;

(9) 剔除有数据缺失的样本。
篮选后本文共获得 16,271 个有效样本，为了消除 离群值的影响, 本文对主要连续变量进行了 1 $29 \%$ 水平 的缩尾处理 (Winsorize)。本文数据预处理软件为 Python, 社会网络指标计算采用社会网络数据分析软件 NetworkX, 统计分析软件为 Stata15. 1。

\section{2. 模型设定与变量说明}

本文采用二元 Logistic 回归法, 借鉴前人（万良 勇、梁雯、左晓宇 [7］９］［10］）的研究设计思路, 以 并购概率代表并购意愿，构建检验模型（1）:

MAt $=\beta 0+\beta 1$ Networkt $-1+\beta 2$ Sizet $-1+$ $\beta 3$ Tobin'qt $-1+\beta 4$ Liqt $-1+\beta 5$ ROAt $-1+$ $\beta 6$ Growtht $-1+\beta 7$ Levt $-1+\beta$ 8Dualt $-1+$ $\beta$ Boardt $-1+\beta 10$ Indept $-1+\beta 11$ Firmage + $\beta 12$ Firstt $-1+\beta 13$ ESHt $-1+\Sigma \beta 14$ Industry + $\Sigma \beta 15$ Year (1)

并购概率 MAt 为公司当年进行并购的可能性, 社会 网络数据、控制变量数据均取并购首次公告前一年数据, 因考虑到公司并购是一个严谨的决策, 有提前评估、接 触、谈判、意向的过程, 需要一定提前量。

\section{3. 被解释变量}

对于研究模型 (1), 被解释变量 MAt 为并购概率, 为 二分类变量, 如果 $\mathrm{i}$ 公司在第 $\mathrm{t}$ 年发生了并购, 取值 为 1 , 未发生并购取值为 0 。

\section{4. 解释变量}

本文采用约束系数指标来衡量结构洞丰富程度。参 考 Burt (2004) 的做法来计算结构洞的相关指标 [11]。 Burt 指标中的结构洞约束数值越大, 代表着企业所处位 置的结构洞越小, 所以, 学者们常用 Burt 所提出的结构 洞约束与 1 的差值来衡量结构洞的丰富程度 $(\mathrm{SHi})$, 即 SHi 越大, 代表着公司所处网络位置的结构洞越丰富。

结构洞约束系数是对节点 $\mathrm{v}$ 在自身已连接于 $\mathrm{v}$ 的邻 居的那些节点中连接的程度的度量, 其中 $\mathrm{N}(\mathrm{v})$ 是 $\mathrm{v}$ 的 邻居的子集, 而 $\mathrm{ell}(\mathrm{v}, \mathrm{w})$ 是 $\mathrm{v}$ 相对于 $\mathrm{w}$ 的局部约束。 计算公式如下:

$$
c(v)=\sum_{w \in N(v) \backslash\langle v\rangle} \ell(v, w)
$$

图 1 约束系数公式, 来源: NetworkX 软件

本文结构洞丰富程度的计算公式如下:

$$
\mathrm{SHi}=1-\mathrm{c}(\mathrm{v})
$$




\section{5. 控制变量}

本文参考已有文献，控制了以下可能会影响公司并 购决策的因素：公司规模（Size）、公司总资产收益率 （ROA）、公司营业收入增长率（Growth）、公司托宾 Q 值 (Tobin`s Q) 、流动能力 ( Liq) 、公司负债率 (Lev)、 公司产权性质 (Property)、董事长总经理是否兼任 (Dua1)、董事会规模 (Board)、独立董事比例 (Indep)、 第一大股东持股比例 (First)、管理层持股比例 (ESH) 等和年度及行业固定效应。

\section{4. 实证结果与分析}

\section{1. 描述性统计分析}

本文对主要变量进行了描述性统计与相关分析（限 于篇幅, 未详细列示）。在样本中, $\mathrm{A}$ 股上市公司有约 64\%的公司没有发生并购。公司网络中心度的均值为 4. 47 , 说明一家公司平均与 4.47 家公司有共享高管的现 象。

\section{2. 公司结构洞丰富程度与并购行为的回归结果}

如表 1 所示, MA 和结构洞丰富程度在 $1 \%$ 水平上显 著正相关, 说明相关上市公司所处的网络位置的丰富程 度可以显著提高上市公司并购的发生概率, 与假设 1 相 符。（限于篇幅, 部分控制变量未详细列示）

\section{表 1 结构洞对并购意愿的 Logit 回归结果}

\begin{tabular}{|l|l|}
\hline 自变量 & MAt \\
\hline SHi & $0.336^{* * *}$ \\
\hline size & $0.084^{* * *}$ \\
\hline lev & 0.050 \\
\hline tobinq & $-0.042^{* *}$ \\
\hline roa & $3.019^{* * *}$ \\
\hline liq & $-0.751^{* * *}$ \\
\hline growth & $0.037 * * *$ \\
\hline esh & $0.654^{* * *}$ \\
\hline first & $-0.598^{* * *}$ \\
\hline board & $-0.033^{* *}$ \\
\hline indep & -0.229 \\
\hline$\ldots$ & $\ldots$ \\
\hline cons & $-1.437 * * *$ \\
\hline Obs. & 12589 \\
\hline Pseudo R2 & 0.033 \\
\hline
\end{tabular}

注: ***、**和*分别代表在 1\%、5\%和 $10 \%$ 的水平上显 著; 来源: 作者汇总 Stata 结果

\section{5. 结论}

从社会网络理论的视角来看, 上市公司在社会网络 中占据的位置会对其并购行为和绩效产生影响。本文从 高层管理团队任职兼任形成的公司网络的视角, 通过对 2008 年至 2017 年间中国上市公司研究发现，上市公司 的结构洞丰富程度会对公司并购行为的产生带来正向推 动作用。这可能是由于当上市公司所处网络位置的结构 洞越丰富时，上市公司将会有更多的机会获取镶嵌于社 会网络中的社会资本与市场信息，而随着上市公司的社 会资本与市场信息不断的累积, 产生了 “控制” 与 “信 息” 方面的竞争优势, 从而对并购产生正向推动作用。

\section{REFERENCES}

[1] Haunschild, P. R. (1994). How much is that company worth?: Interorganizational relationships, uncertainty, and acquisition premiums. Administrative Science Quarterly, 391-411.

[2] Chen Yunsen, \& Xie Deren. (2012). Director Network, Independent Director Governance and Executive Incentives. Financial Research, 2, 168-182.

[3] Burt, R. S. (1992). The social structure of competition.

[4] Cai, Y., \& Sevilir, M. (2012). Board connections and M\&A transactions. Journal of Financial Economics, 103(2), 327-349.

[5] Boussard, V., Godechot, O., \& Woloszko, N. (2016). How to make a deal: The role of rankings and personal ties in creating trust in the M\&A market.

[6] Shipilov, A. V., \& Li, S. X. (2008). Can you have your cake and eat it too? Structural holes' influence on status accumulation and market performance in collaborative networks. Administrative Science Quarterly, 53(1), 73-108

[7] Wan Liangyong, Zheng Xiaoling. Structural hole characteristics of director networks and corporate mergers and acquisitions [J]. Accounting Research, 2014, (5).

[8] Tian Gaoliang, Han Jie, \& Li Liu Chuang. (2013). Chain Directors and M \& A Performance: Empirical Evidence from Chinese A-Share Listed Companies. Nankai Management Review, 16 (6), 112-122.

[9] Liang Wen, Liu Shulian, \& Li Jihan. (2018). Network location, director experience and $\mathrm{M} \& \mathrm{~A}$. Journal of Shanxi University of Finance and Economics, (7), 8 . 
[10] Zuo Xiaoyu, \& Sun Qian. (2017). Director Network, Corporate Centrality and M \& A. Investment Research, 36 (2), 4-16.

[11] Burt R S. Structural holes and good ideas[J]. American journal of sociology, 2004, 110(2): 349-399. 